\title{
Europe targets Mercury for future mission
}

Paris. The European Space Agency (ESA) announced this week that its priorities in space science early next century will probably include a mission to the planet Mercury and an interferometric study of the distances, motions and luminosities of tens of millions of stars in the Milky Way.

The two missions were chosen from 110 proposals submitted by European scientists - and 14 from scientists in the United States - as candidates for 'cornerstone' missions in ESA's planned Horizon 2000plus space science programme. This will run for ten years from 2006 , and will follow the current Horizon 2000 programme.

The quality and cost-effectiveness of ESA's 'Horizon' programmes have been widely acclaimed. Many attribute their success to the funding stability they enjoy; ESA's 13 member states are obliged to contribute a fixed proportion of their gross national product, while future funding levels are agreed in five-year chunks. This year, ESA will spend ECU381 million (US\$472 million) on science, about one-tenth of its total budget.

'Cornerstone' missions are each budgeted to cost around ECU625 million. The Mercury mission would probably include an orbiting observatory and also a landing craft. According to Roger Bonnet, head of science at ESA, the US National Aeronautics and Space Administration (NASA) has offered to take part if ESA decides to go ahead. As a result, the mission could become a European-led international effort.

The astronomy cornerstone would use interferometry to survey stars in the Milky Way, to search for Jupiter-sized planets and signs of life - around stars in our Galaxy, and to test the theory of general relativity. Instruments on the craft would increase the resolution of current satellites by one to two orders-of-magnitude, giving them a capacity to resolve from Earth objects two millimetres apart on the Moon.

In what would be ESA's first venture into fundamental physics, the space agency is also considering a cornerstone mission to detect gravitational waves emitted, for example, from black holes, binary stars and the early Universe. Such a mission would also be technologically daunting, requiring the launch of six satellites millions of kilometres apart, and keeping them connected by laser beams.

But much depends on funding. Bonnet points out that, although the first two missions cornerstones could be achieved within a static budget, one to detect gravitational waves would require a 4 to 5 per cent increase in ESA's science budget between 2000 and 2005. ESA will carry out technological studies before deciding whether to request further funding.

In addition to the big cornerstone missions, Horizon programmes also include several 'medium-sized' missions, budgeted at around ECU345 million, chosen by in open competition combined with peer review. One candidate for Horizon 2000-plus is a mission to Mars. ESA would have proposed this as a cornerstone, says Bonnet, if the United States, Japan and Russia did not already have similar plans.

ESA officials say that solar missions should also be included as medium-sized missions in Horizon 2000-plus, although specific details have not yet been decided. Looking beyond Horizon 2000 plus, the survey committee has recommended that Europe build on its lead in X-ray, gammaray and infrared astronomy.

ESA's science programme committee is

\section{IMAGE UNAVAILABLE FOR COPYRIGHT REASONS}

The Red Planet: ESA's new priority.

expected to approve the proposals next month. Final approval will have to wait until the meeting of Europe's space ministers late next year, which is expected to look closely at the balance between science missions and the more expensive (and increasingly criticized) efforts in manned spaceflight. Ministers will not need to commit money to Horizon 2000-plus until 2000, says Bonnet, as preliminary work can be done within existing budget allocations.

Declan Butler

\section{Germany holds up bioethics treaty}

Paris. The parliamentary committee of the 32-member Council of Europe last week postponed a vote on the draft European Convention on Bioethics after opposition from Germany. German representatives on the committee argued that the text should be more restrictive and less ambiguous.

In particular, the German government wants the council to support tighter regulations on the use of human embryos in research. Its opposition has highlighted the difficulty in achieving international consensus on bioethics issues; the provisions of a draft released in July already amounted to

\section{Berlin protestors condemned by minister}

Munich. Scientists at east Berlin's large teaching hospital, the Charité, upset at a planned merger with west Berlin's Rudolf Virchow clinic, have been sharply criticized by Berlin's ministry of research for bringing the issue into the recent general election campaign.

A group of scientists representing nonprofessorial scientific staff leafleted the city last week, calling on voters not to vote in last Sunday's election for the two major parties in Berlin's grand coalition, the Social Democrat Party (SPD) and the Christian Democrat Union (CDU), because of the damage they claim that they have caused the hospital.

Manfred Erhardt, Berlin's research minister, condemned the attempt to use the hospital's problems in an election campaign as "irresponsible and damaging to the Charité". But supporters of the scientists claim they had been left with no option, as no officials appeared to be listening to their complaints. Alison Abbott little more than the lowest common denominator (see Nature 370, 3; 1994).

But Germany feels that a provision of the draft convention forbidding research on human embryos more than 14 days old is insufficient. It also said that provisions on handicapped people could open the way to their use as research subjects.

Supporters of the convention claim that it provides an umbrella of fundamental principles, and that member states can implement stricter legislation if they choose.

German resistance stems partly from the fact that the bioethics convention became a political issue in the run-up to the general elections last Sunday (16 October), largely as a result of a general sensitivity in Germany to issues related to genetics and human experiments. All the political parties had promised they would reject the text on the grounds that it did not go far enough.

The committee had hoped to approve the text ahead of the next meeting in January 1995. It will now seek a compromise with Germany to vote in at the January meeting. If this goes to plan, the convention - which is now two years behind schedule - may be presented to member states for ratification in March next year.

D. B. 